\title{
Phyto-oestrogens affect fertilisation and embryo development in vitro in sheep
}

\begin{abstract}
Phyto-oestrogens such as isoflavones are natural compounds that can profoundly affect reproductive function. In the present study, we tested whether including isoflavone compounds (genistein, biochanin A, formononetin) in the maturation medium would affect the outcomes for ovine oocytes in vitro. Each isoflavone compound was evaluated at five concentrations ( 0 , $2.5,5,10,25 \mu \mathrm{gmL}-1)$ and the entire protocol was repeated four times. Cumulus-oocyte complexes were randomly allocated to the treatments, then fertilised and cultured in vitro.

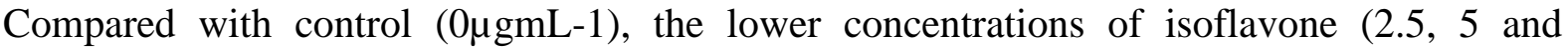
$10 \mu \mathrm{gmL}-1)$ had no detectable effect on the rates of cleavage or embryo development, or on embryo total cell counts (TCC). However, the highest concentration $(25 \mu \mathrm{gmL}-1)$ of all three isoflavones exerted a variety of effects $(\mathrm{P}<0.05)$ : genistein decreased cleavage rate, blastocyst rate and blastocyst efficiency (blastocysts produced per 100 oocytes); biochanin A decreased cleavage rate and blastocyst efficiency; and formononetin decreased blastocyst rate and blastocyst efficiency. Biochanin A $(25 \mu \mathrm{gmL}-1)$ reduced embryo TCC specifically at the hatched blastocyst stage $(\mathrm{P}<0.05)$. We conclude that the presence of isoflavones at $25 \mu \mathrm{gmL}-1$ during IVM decreases the cleavage rate and inhibits blastocyst hatching.
\end{abstract}

Keyword: Biochanin A; Blastocyst; Formononetin; Genistein; Oocytes 